\title{
Overview of the LDL receptor: relevance to cholesterol metabolism and future approaches for the treatment of coronary heart disease
}

This article was published in the following Dove Press journal:

Journal of Receptor, Ligand and Channel Research

15 December 2009

Number of times this article has been viewed

\section{William R Lagor \\ John S Millar}

Department of Pharmacology, Institute for Translational Research and Therapeutics, University of Pennsylvania, Philadelphia, PA, USA
Correspondence: John S Millar University of Pennsylvania, 652 BRB II/III, 42I Curie Blvd, Philadelphia, PA 19104, USA

Tel + I 2158980638

Fax (215) 5738606

Email jsmillar@mail.med.upenn.edu

\begin{abstract}
Since the discovery of the low-density lipoprotein (LDL) receptor over 30 years ago manipulation of its activity has been pursued through dietary and pharmacological means with the goal of reducing LDL cholesterol levels. This has proven to be one of the most widely implemented and beneficial interventions to reduce LDL cholesterol levels and the incidence of cardiovascular death. This review provides an overview of the LDL receptor and its role in regulating whole body cholesterol homeostasis and LDL cholesterol levels. New findings on regulation of the LDL receptor are discussed and how they can be used to develop novel therapeutics to reduce cardiovascular risk.
\end{abstract}

Keywords: VLDL, statins, pcsk9, cardiovascular disease

\section{Overview of the LDL receptor}

Cholesterol is an essential component of cellular membranes, as well as an essential substrate for steroid hormones and bile acid synthesis. However, cholesterol is toxic when accumulated in excess in cellular membranes ${ }^{1}$ and elaborate pathways have evolved to control its synthesis, uptake and storage. ${ }^{2}$ Cells can obtain cholesterol either from de novo synthesis, or uptake from circulating lipoproteins. The low-density lipoprotein (LDL) receptor (LDLR) is the primary pathway for removal of cholesterol from the circulation, ${ }^{3}$ and its activity is meticulously governed by intracellular cholesterol levels. ${ }^{4}$ The discovery of the LDLR had its roots in the study of the molecular mechanism responsible for familial hypercholesterolemia (FH), an autosomal dominant metabolic disorder in which patients present with plasma cholesterol levels ranging from $300 \mathrm{mg} / \mathrm{dL}$ to as high as $1500 \mathrm{mg} / \mathrm{dL} .{ }^{5}$ Patients with $\mathrm{FH}$ are at increased risk of cardiovascular disease and those with the highest cholesterol levels often have advanced atherosclerosis before 10 years of age. ${ }^{6}$

FH was first recognized as a genetic disorder over 70 years ago when it was observed that extreme hypercholesterolemia that was accompanied by xanthomas (cholesterol deposits in tendons and skin) and angina was an inherited disorder. ${ }^{7}$ In the early 1970s Goldstein and Brown demonstrated that fibroblasts isolated from patients with homozygous FH had defective feedback inhibition of the enzyme 3-hydroxy-3-methylglutaryl CoA reductase (HMGCR), the rate-limiting enzyme for cholesterol synthesis. ${ }^{8}$ In 1974 they were able to demonstrate the existence of a LDLR by showing that fibroblasts from patients with homozygous FH were unable to bind LDL at the cell surface. ${ }^{9}$ The following year they showed that the LDLR regulated HMGCR activity by controlling the cellular uptake of exogenous cholesterol ${ }^{10}$ submit your manuscript $\mid$ www.dovepress.com

Dovepress 
rather than through signaling via a second messenger as they originally hypothesized. ${ }^{5}$ The LDLR internalized LDL from plasma leading to release of cholesterol into the cell which appeared to be responsible for the feedback inhibition of HMGCR that they had observed in their earlier studies. The LDLR protein was isolated and sequenced in $1982^{11}$ and the gene cloned in $1985 .{ }^{12}$ Site-directed mutagenesis of the LDLR gene and analysis of natural mutants led to detailed identification and characterization of the functional domains of the receptor. ${ }^{13}$ Over 1000 naturally occurring mutations in the LDLR gene have been reported to date with varying degrees of clinical severity depending on the site of the mutation. ${ }^{6,14}$

In the general population reduced LDLR activity frequently contributes to hypercholesterolemia which over time, contributes to the development of atherosclerosis. Knowledge gained from studies about LDLR function, structure and regulation has led to the development of drugs and recommendations to reduce hypercholesterolemia in the population as a whole. This had led to reduced rates of cardiovascular disease and, in recent years, strategies that can even reverse atherosclerosis through manipulation of LDLR activity. Recent developments regarding the LDLR hold promise as future therapies that will continue this trend.

\section{LDL receptor structure}

The LDLR is a transmembrane protein that is 839 amino acids in length that can broadly be divided into 5 domains (Figure 1). Based on structural analysis, the functions associated with these domains in the protein have been identified. Each of these will be discussed individually.

\section{LDLR repeat domain}

Also referred to as the LDLR type A repeat domain. This area has been shown to be responsible for ligand binding of the receptor; the known ligands being apolipoprotein (apo) B100 on low density lipoprotein ${ }^{15}$ and lipoproteins containing apoE. ${ }^{16}$ The LDLR repeat domain consists of seven homologous repeats that are each approximately 40 amino acids in length. ${ }^{17}$ Within each domain are regions of acidic amino acids that provide the negative charge for the ionic interaction necessary for binding the basic amino acids within the LDLR binding domain of apoB $100^{18}$ and $a p o E^{19}$ ligands. It has been known for some time that an ionic interaction with calcium is required for proper alignment of acidic residues within the LDLR repeat domain and that ligand binding does not occur in the presence of EDTA. ${ }^{20}$

\section{EGF repeat domain}

The epidermal growth factor (EGF) receptor homology domain consists of three repeated sequences that are homologous (35\% homology) to the EGF receptor. ${ }^{21}$ The first two EGF domains are linked to the third by a short sub-domain of 6 short amino acid repeats (YWTD) that form a six-bladed $\beta$-propeller. ${ }^{22}$ The $\beta$-propeller may interact with repeats 4 and 5 in the LDLR repeat domain and contributes to ligand release from the receptor following internalization once it encounters the low $\mathrm{pH}$ of the lysosome. ${ }^{23}$

\section{O-linked glycosylation domain}

This is a 58 amino acid section that is rich in serine and thought to serve as a spacer region between the EGF Repeat domain and the plasma membrane. ${ }^{21}$ The O-linked sugars may prevent proteolytic cleavage of the extracellular domain while it is situated on the plasma membrane. ${ }^{24}$ Mutation/ deletion of this region has no effect on ligand binding. ${ }^{25}$

\section{Transmembrane domain}

This consists of an approximate 25 amino acid section enriched in hydrophobic amino acids that serves to anchor the LDLR within the plasma membrane. ${ }^{13}$ A mutant that is truncated within this region has a reduced capacity to remain attached to the plasma membrane and is also found free in plasma. ${ }^{26}$

\section{Cytoplasmic domain}

This consists of a 56 amino acid section that contains an NPXY sequence that binds to ARH1, ${ }^{27}$ (discussed in detail below). This domain also contains a glycine at position 823 that is required for the proper sorting of the receptor to the plasma membrane following synthesis. ${ }^{28}$

\section{ApoB 100 and apoE-containing ligands}

Both cholesterol and triglyceride are required for normal metabolism. Their distribution to tissues where they are needed is controlled primarily by the liver. Since triglyceride and cholesterol are lipophilic substances, they are packaged by the liver as part of a water soluble lipoprotein complex known as very low-density lipoprotein VLDL. These lipoprotein particles are secreted into plasma where their lipids are delivered to peripheral tissues. The VLDL particle is a lipid/protein complex which consists of a spherical particle containing phospholipid and free cholesterol on the surface and triglyceride and cholesteryl ester in the core of the particle. The exact structure of the main protein component of VLDL, apoB100, is not precisely known due to difficulties in determining the structure of proteins within a lipid matrix. However, current models, based on a compilation of 


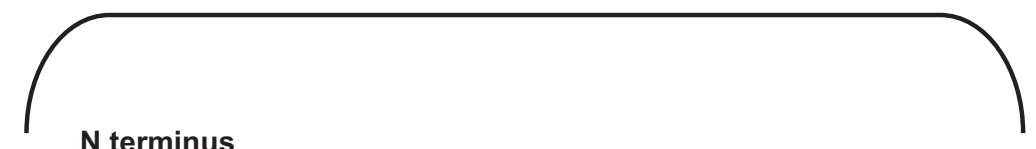

\section{R1}

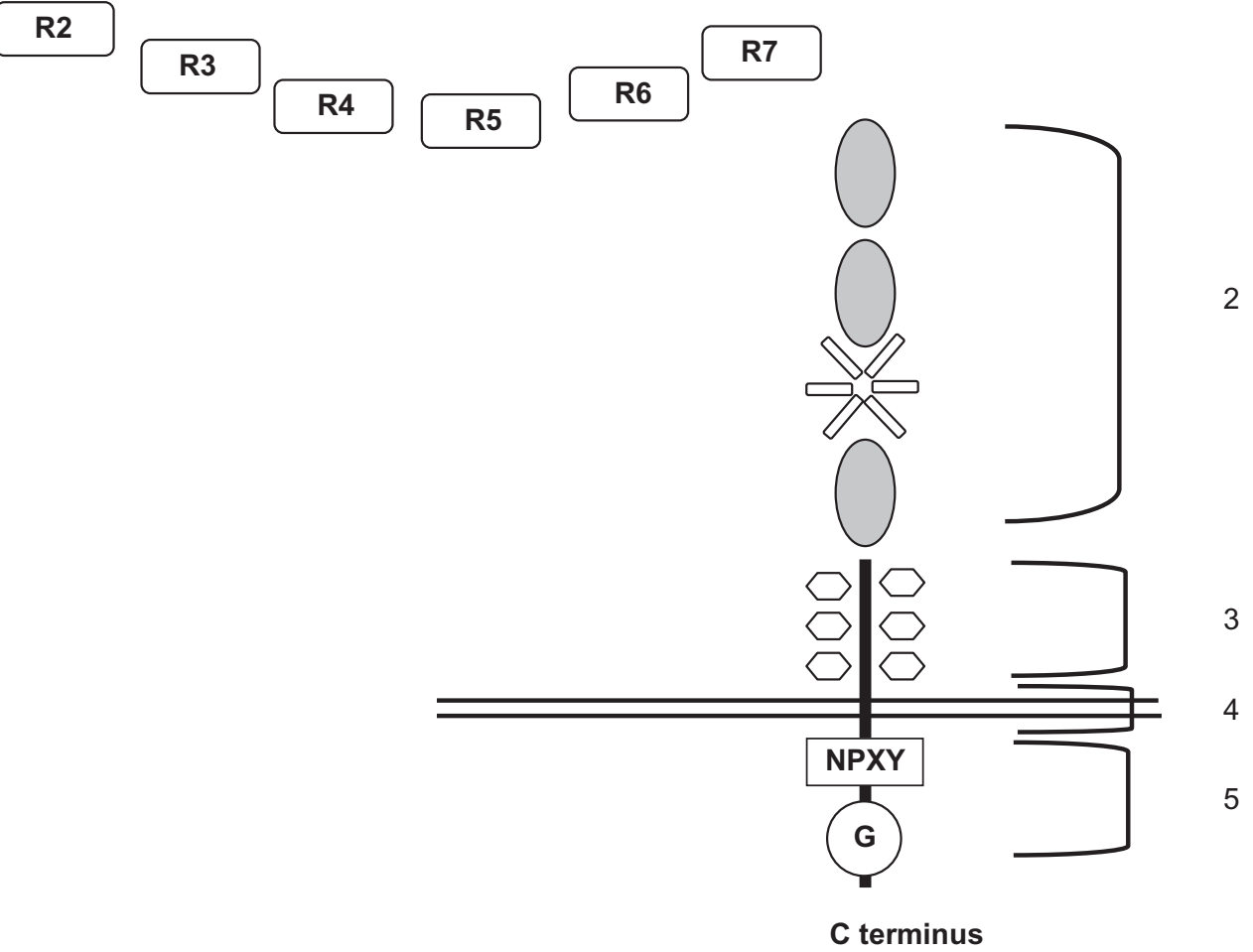

Figure I A schematic diagram illustrating the 5 domains of the low-density lipoprotein receptor (LDLR). I) LDLR type A repeat domains 2) epidermal growth factor (EGF) receptor homology domain containing the $\beta$-propeller subdomain 3) O-linked glycosylation domain 4) transmembrane domain 5) cytoplasmic domain containing NPXY sequence that binds to $\mathrm{ARHI}$ and glycine at position 823 that is required for the proper sorting of the receptor following synthesis.

available structural data, suggest that the apoB100 protein forms a band that encircles the VLDL particle. ${ }^{29}$ As VLDLs circulate their triglyceride content is reduced through the action lipases in plasma. ${ }^{30}$ In addition, these particles accept cholesterol from high density lipoproteins, a process mediated by cholesteryl ester transfer protein (CETP). ${ }^{31}$ The result of this metabolic activity is that a portion ( $50 \%$ to $90 \%$ ) of VLDL is eventually converted to a cholesterol-rich LDL particle that is smaller and denser than VLDL.

\section{General overview}

The LDLR has been tested against all classes of lipoproteins and has been demonstrated to bind to lipoproteins containing apoE $^{16}$ as well as apoB in LDL. ${ }^{32}$ The receptor is most active against beta VLDL (containing apoE), intermediate-density lipoprotein (IDL), and low-density lipoprotein (LDL) ${ }^{32}$ and large high-density lipoprotein (HDL) containing apoE. ${ }^{33}$
The interaction between receptor and ligand is electrostatic with acidic residues in the LDLR binding domain interacting with basic residues on the apoB100 and apoE ligand, ${ }^{18,19}$ While the exact nature of this interaction hasn't been fully characterized due to difficulties inherent in crystallizing lipoproteins, structural studies have allowed construction of a model for receptor-ligand interaction in which properly aligned acidic amino acid residues within the LDLR bind to exposed basic residues on apoB and apoE on lipoproteins. ${ }^{23}$

\section{Factors that influence LDL receptor abundance}

Rare mutations have been described that result in absent or defective LDL binding to the LDLR resulting in severe $\mathrm{FH} .{ }^{6}$ However, most cases of hypercholesterolemia seen in the general population are likely due to reduced LDL uptake from plasma either resulting from reduced LDLR activity or to 
suboptimal binding of LDL in the presence of normal receptor activity. ${ }^{34}$ Effective pharmacologic treatment with statins results in enhanced LDL clearance from plasma. ${ }^{35}$ The next section will describe the molecular mechanisms responsible for these changes that lead to lower LDL levels (Figure 3).

\section{SREBPs}

Sterol regulatory element binding proteins (SREBPs) are membrane bound transcription factors that are synthesized as inactive precursors in the endoplasmic reticulum. When sterol levels in the endoplasmic reticulum are low, ${ }^{36}$ a series of molecular events takes place that results in the escort of SREBPs to the Golgi apparatus where they are processed to release their active $\mathrm{N}$-terminal fragments. ${ }^{37}$ The $\mathrm{N}$-terminal fragment consists of a basic helix-loop-helix transcription factor that rapidly translocates to the nucleus to activate transcription of target genes. ${ }^{38}$ There are two genes encoding SREBPs, giving rise to three different isoforms: SREBP-1a, SREBP-1c and SREBP-2. 39,40 The SREBP-1 gene encodes the SREBP-1a and SREBP-1c isoforms that activate genes primarily related to fatty acid metabolism, ${ }^{41}$ while SREBP-2 preferentially targets genes related to cholesterol biosynthesis and uptake including HMGCR and the LDLR. ${ }^{42}$ While the LDLR promoter can be activated by both SREBP-1 and 2 in cultured cells, evidence from transgenic and knockout mice suggests that SREBP-2 plays a more dominant role in vivo. ${ }^{43}$ The N-terminal fragment of SREBP-2 binds to a specific sterol regulatory element (SRE) in the LDLR promoter. ${ }^{40}$ Transcriptional activation of $L D L R$ by SREBP-2 requires the cooperative action of the transcription factor Sp1 at neighboring sites. In the absence of binding of SREBP-2, the LDLR promoter can be silenced through the repressive action of KLF13. ${ }^{44}$ SREBP-2-mediated activation of $L D L R$ transcription is a direct way for the cell to address cholesterol depletion. Likewise, sufficient sterol levels in the ER membrane prevent SREBP-2 transport to the Golgi for cleavage, suppressing basal $L D L R$ transcription and thereby limiting excessive cholesterol uptake.

\section{$\mathrm{ARH}$}

Autosomal recessive hypercholesterolemia (ARH) resulting from deficiency of the ARH protein (also known as low density lipoprotein receptor adaptor protein 1, LDLRAP1) is an adaptor protein that binds to the cytoplasmic tail of the LDLR and is required for its efficient internalization in the liver. ${ }^{45}$ The $A R H$ gene was initially identified through linkage analysis of affected pedigrees and careful sequencing of candidate genes in the associated region on the short arm of chromosome $1 .{ }^{46}$ Family members who are heterozygous for deleterious mutations in ARH have normal serum cholesterol levels, presumably due to sufficient ARH activity from the normal allele. However, subjects with homozygous ARH deficiency present with severe hypercholesterolemia comparable to what is seen in $\mathrm{FH} .{ }^{47}$ Interestingly, fibroblasts from subjects homozygous for ARH deficiency display only a modest impairment in LDL uptake. ${ }^{48}$ In contrast, LDL clearance from plasma is greatly reduced, suggesting a specific effect of ARH on hepatic LDLR levels. ${ }^{49,50} \mathrm{ARH}$ is highly expressed in the liver, ${ }^{27}$ and is believed to play an important role in coupling the LDLR to the endocytic machinery in this tissue.

ARH encodes a small protein of 308 amino acids consisting of a 40 residue $\mathrm{N}$-terminus, followed by a phosphotyrosine binding (PTB) domain and a clathrin box consensus sequence. The PTB domain of ARH binds to a conserved NPXY motif on the cytoplasmic tail of the LDLR. An LLDLE pentapeptide sequence in ARH mediates its high affinity binding to clathrin, ${ }^{51}$ the defining structural component of the clathrin coated pit in which the LDLR resides on the plasma membrane. ARH also specifically interacts with the $\beta_{2}$-adaptin subunit of AP-2, ${ }^{51}$ a protein complex responsible for endocyctosis of clathrin coated vesicles. The function of ARH appears to be linking the LDLR receptor to the endocytic machinery of the cell. Targeted deletion of the $A R H$ gene in mice results in dramatically higher LDL cholesterol levels in plasma when the animals are placed on a high-cholesterol diet due to impaired clearance of LDL from the plasma resulting from defective endocytosis of LDL particles. ${ }^{52}$ Although hepatic LDLR protein levels are normal in these animals, a much greater proportion of the receptor is found trapped on the sinusoidal surface of hepatocytes. ARH must bind to the cytoplasmic tail of the LDLR along with either clathrin or AP-2 to promote internalization of LDL in these cells. ${ }^{53}$ In non-hepatic cell types, the related Dab2 phosphotyrosine binding domain protein functionally substitutes for ARH by catalyzing the clustering of LDLR into clathrin coated pits. ${ }^{54}$ This explains why LDLR activity is normal in extra-hepatic cells, such as fibroblasts, from ARH deficient animals and humans. However, there are still many unanswered questions regarding the sequence of events by which ARH facilitates LDL uptake at the molecular level.

\section{Idol}

Inducible degrader of the LDLR (Idol) is a protein that has recently been found to be capable of promoting degradation 
of the LDLR following translation in the ER. LDLR protein levels in hepatic cell lines were shown to be reduced upon treatment with an agonist to the hydroxysterol-sensitive nuclear receptor LXR although the mechanism for this was unknown. LXR is present in two isoforms, $\alpha$ and $\beta$, that have different tissue expression patterns but are activated similarly by LXR agonists. Zelcer and colleagues utilized microarray profiling of LXR agonist treated HepG2 cells to identify candidate genes that were responsible for reduced LDLR expression. ${ }^{55}$ They identified a putative E3 ubiquitin ligase that they named Idol (previously known as Mylip or Mir). ${ }^{56}$ Idol contains a band 4.1 and Ezrin/Radixin/Moesin homology (FERM) domain that is known to interact with cytoplasmic regions of transmembrane proteins. In addition, Idol has a RING domain typical of E3 ubiquitin ligases on its C-terminus. Overexpression of Idol in cultured cells resulted in increased ubiquitination and degradation of LDLR protein following translation. Degradation of the receptor required the presence of a lysine (K20) and a cysteine (C29) in the intracellular domain of LDLR, presumably sites of ubiquitination by Idol. The authors further demonstrated that shRNA knockdown of Idol increased LDLR expression in MEF and McR-H7777 cells. Accordingly, knockdown of Idol abrogated the LXR-mediated reduction in LDLR protein levels in these cell lines. In mice, adenoviral-mediated overexpression of Idol virtually eliminated hepatic LDLR protein and robustly increased plasma LDL cholesterol levels in these animals. Strangely, treatment of mice with an LXR agonist reduced LDLR in several peripheral tissues, but not the liver, suggesting other regulatory pathways may predominate in this tissue. Conversely, $\mathrm{LXR} \alpha / \beta$ knockout mice had slightly higher LDLR protein levels in the liver, which would be consistent with reduced Idol-mediated turnover of the LDLR. This recent report suggests that Idol may play a novel role in regulating LDLR turnover, and thereby plays an important role in controlling plasma and cellular cholesterol levels and is a potential pharmacologic target to enhance LDLR activity in liver leading to reduced LDL cholesterol levels in plasma.

\section{Thyroid hormone}

Thyroid hormone regulates basal metabolic rate and circulates in two forms in plasma, triiodothyronine (T3) and thyroxine (T4). These bind to thyroid hormone receptor (TR), which exists as two isoforms, TR- $\alpha$ and TR- $\beta$, which form an active complex that promotes transcription of target genes. As early as 1979, Chait, and colleagues noted that uptake and degradation of LDL by cultured fibroblasts from normal subjects was enhanced by the addition of T3. ${ }^{57}$ Hyperthyroid rats are known to have enhanced LDL clearance while that from hypothyroid rats is dramatically impaired. ${ }^{58}$ Similarly, humans with hypothyroidism have increased levels of LDL in plasma due to markedly impaired LDL clearance from plasma. Administration of T4 reduces LDL levels presumably due to enhanced LDL clearance. ${ }^{59}$ The effect of thyroid hormone on LDL cholesterol levels is believed to be due primarily to increases in LDLR expression. In rats, thyroid hormone treatment markedly increases LDLR mRNA and protein levels. ${ }^{60-62}$ The increase in LDLR mRNA is not due to alterations in message stability, ${ }^{63}$ but rather an increased rate of LDLR transcription. ${ }^{64}$

Recent work has shed light on the mechanisms by which thyroid hormone activates LDLR transcription. Bakker and colleagues found that the human LDLR promoter can be activated by T3 in HepG2 cells. ${ }^{65}$ A functional thyroid responsive element (TRE) was identified between -687 and -160 bp upstream of the ATG start codon, although the existence and/or precise locations of TR binding sites were not delineated. Shin and colleagues discovered that SREBP-2, a potent activator of LDLR transcription, ${ }^{40}$ is itself subject to transcriptional regulation by thyroid hormone. ${ }^{66}$ The SREBP-2 promoter contains a direct repeat 4 (DR4) site that binds TR- $\alpha$ and TR- $\beta$. They demonstrated that the SREBP-2 promoter can be activated by thyroid hormone, and mature SREBP-2 protein levels do vary with thyroid hormone status in mice. The authors suggested that T3 induction of SREBP-2 expression may be sufficient to explain thyroid hormone activation of the LDLR, ${ }^{66}$ although more direct effects were not ruled out. To test this hypothesis, Lopez and colleagues examined the rat $L D L R$ promoter ${ }^{67}$ and identified functional TREs in the promoter that are capable of binding TR- $\alpha$ and TR- $\beta$ in vitro. Combined mutagenesis of these TREs at -612 and -156 in the rat $L D L R$ promoter completely abolished T3-mediated activation, consistent with the previously reported elements in the human $L D L R$ promoter. ${ }^{65}$ In these studies LDLR activation was rapid (1-2 hours after T3), and preceded a slower rise in SREBP-2 protein levels (several days). Taken together, the data suggest LDLR is a direct TR- $\beta$ target gene, and that subsequent induction of SREBP-2 expression by thyroid hormone likely augments this effect. Since TR- $\beta$ is the major TR isoform in liver, this finding has clinically relevant implications. Strategies to improve thyroid hormone responsiveness would be expected to increase hepatic LDLR protein and activity. This would likely provide significant therapeutic benefit to hypercholesterolemic subjects with underlying hypothyroidism. 


\section{Factors that influence lipoprotein binding and affinity to the LDL receptor \\ ApoBI00 conformation}

While individual VLDL, IDL or LDL particles contain a single apoB100 peptide, each of these particles is known to have differing affinities to the LDLR with IDL/LDL > VLDL. ${ }^{68}$ Even within each lipoprotein class, lipoprotein particles that differ in lipid composition and size display different affinities for the LDLR. ${ }^{69}$ The reason for the differences in affinity of these different types of apoB-containing lipoproteins became apparent when the ribbon and bow model of apoB within LDL was developed..$^{29}$ This model was constructed by computing the relative locations of monoclonal antibodies to different epitopes of apoB on LDL. The model has the first $89 \%$ of apoB encircle LDL with the C-terminal $11 \%$ folding back over the apoB100 peptide near the purported LDLR binding region between amino acids 3359-3369 of apoB (Figure 2). Boren and colleagues performed mutation studies on apoB and determined that the region containing amino acids 3359-3369 was responsible for binding to the LDLR. ${ }^{70}$ Earlier reports showing the importance of a naturally-occurring mutation in amino acid 3500 of apoB in binding to the LDL receptor. ${ }^{71}$ This was originally thought to be a mutation within the LDLR binding domain of apoB. However, Boren and colleagues concluded that the residue at position 3500 forms a bridge with the $\mathrm{C}$-terminal portion of apoB that would otherwise interfere with receptor binding to the region at amino acids 3359-3369. Thus the C-terminus of apoB crosses over the region near the receptor binding region and can regulate interaction between apoB and the LDLR. These differences are likely sensitive to subtle changes in the conformation of the LDLR-binding domain in apoB100
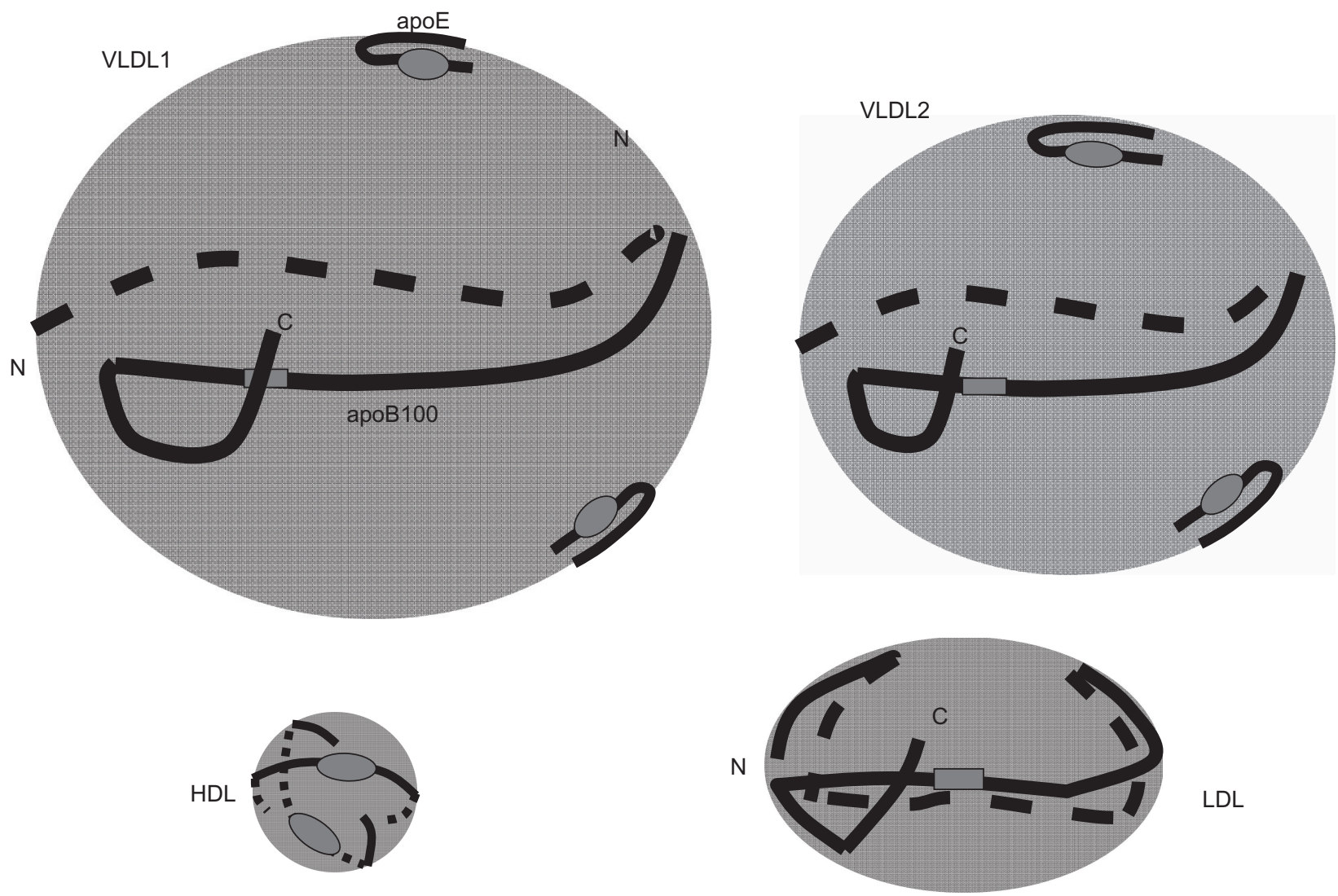

LDL

Figure 2 General models illustrating low-density lipoprotein receptor (LDLR) binding domains on apoBI00 (shaded rectangles) and apoE (shaded ovals) in very low-density lipoprotein (VLDL) I (large, triglyceride-rich VLDL), VLDL2 (smaller, triglyceride-depleted VLD), low density lipoprotein (LDL) and high density lipoprotein (HDL). Each lipoprotein particle consists of a lipid sphere (shaded circles) consisting of a cholesteryl ester/triglyceride core surrounded by a monolayer of phospholipid/free cholesterol. ApoBI00, encircles VLDLI, VLDL2 and LDL, is represented by the black line containing the shaded rectangle and is a structural component of these lipoproteins. ApoE is represented by the smaller black line containing the shaded oval. Dashed portions represent parts of each line that are located on the back of the particle. On VLDLI, the C-terminal "bow" on apoB crosses over the LDLR binding domain. These lipoproteins require apoE for uptake by the LDLR. Once VLDLI is depleted of sufficient triglyceride, the LDLR binding domain is exposed allowing apoBI00-mediated binding to the LDLR (VLDL2, and LDL). HDL contains no apoBI00 but may contain apoE. Lipid-enriched HDL containing apoE are able to bind to the LDLR.

Abbreviations: $\mathrm{N}, \mathrm{N}$-terminus; $\mathrm{C}, \mathrm{C}$-terminus. 
due to size and/or local lipid environment of the apoB100 peptide. This would explain the in vivo observations by Vega and Grundy who showed that approximately $30 \%$ of a small group of patients with primary hypercholesterolemia have defective LDL clearance despite normal receptor activity. ${ }^{34}$ Among LDL subspecies it has been demonstrated that the affinity of LDL for the LDLR is associated with the size of an LDL particle. Small LDL particles have lower affinity for the LDLR while large LDL has a higher affinity. ${ }^{72}$

The ribbon and bow model of how apoB100 is positioned on lipoproteins provides an explanation of the difference in affinity for different lipoprotein species and subspecies with the LDLR. VLDL can be subdivided in to large, triglyceriderich particles (VLDL1) and smaller particles (VLDL2) that contain more cholesterol and less triglyceride than VLDL1. VLDL1 are thought to have the LDLR binding region on apoB obscured by the C-terminal portion of the protein. ${ }^{70}$ These large, triglyceride-rich lipoproteins are entirely dependent on apoE for binding to the LDLR. ${ }^{73}$ As VLDL loses triglyceride following delipidation by lipoprotein lipase the lipoprotein particle becomes smaller with the conformation of apoB changing to expose portions of the LDLR-binding domain. This finding may explain the apparent discrepancy found when comparing the results of studies examining the role of the LDLR in regulating the production (secretion) rate of apoB100 in in vivo ${ }^{74,75}$ and in vitro ${ }^{76,77}$ studies. Twisk, and colleagues, reported that the LDLR reduced apoB secretion in studies conducted in cultured cells that secrete apoB100-containing lipoproteins. ${ }^{76}$ In contrast, studies conducted in vivo have found that there's no effect of the LDLR on regulating the production rate of apoB. ${ }^{74,75}$ Since VLDL particles secreted in vivo are larger and more triglyceride rich than those secreted in vitro it is likely that VLDL that are synthesized in vivo would not bind to the LDLR within the secretory pathway whereas those produced in vitro would bind to the LDLR and be targeted for pre-secretory degradation.

\section{ApoE content}

ApoE is an apolipoprotein found on most lipoprotein particles, especially VLDL and HDL that have been demonstrated to bind to the LDLR. There is in vivo evidence that VLDL that are poor in apoE content have an increased probability of being converted to LDL while VLDL enriched with apoE have an increased probability of being removed from plasma by the LDLR. ${ }^{78}$ ApoE with a point mutation in the LDLR binding domain tends to accumulate in plasma and promote atherosclerosis. HDL is a lipoprotein that does not contain apoB but has a cholesterol-rich apoE-containing subfraction that is rapidly cleared from plasma and thought to have an anti-atherogenic role.

\section{ApoC-III}

While apoE has been demonstrated to enhance the binding of lipoproteins to the LDLR, a second exchangeable apolipoprotein, apoC-III has been shown to inhibit the binding of lipoproteins to the LDLR. This inhibitory effect may be due to a direct interaction with the LA4 domain of the LDLR. ${ }^{79}$ Interestingly, apoC-III also inhibits the action of lipoprotein lipase and has been implicated as a causal factor in the development of familial combined hyperlipidemia where both plasma triglyceride levels and LDL cholesterol levels are increased. Carriers of rare null mutation in the apoC-III gene have apoC-III levels in plasma that are half the normal concentration. ${ }^{80}$ These individuals have lower than normal levels of LDL cholesterol and triglyceride.

\section{PCSK 9}

Proprotein convertase subtilisin-like kexin type 9 (PCSK9) is a secreted protease that binds to and promotes degradation of the LDLR protein. Gain-of-function mutations in PCSK9 that enhance its interaction with the LDLR result in markedly higher LDL-C levels in humans. ${ }^{81}$ Conversely, rare loss-of-function mutations in PCSK9 yield dramatically lower LDL cholesterol levels. ${ }^{82}$ PCSK9 is highly expressed in the liver where it is synthesized as an inactive zymogen that undergoes autocatalytic cleavage in the Golgi apparatus to release the prodomain. ${ }^{83}$ The prodomain remains associated with the mature PCSK9 peptide after cleavage and assists in escorting PCSK9 through the secretory pathway ${ }^{83}$ masking catalytic triad of PCSK9 and preventing potential substrates from being cleaved. Once outside the cell, secreted PCSK9 binds specifically to the extracellular EGF-A domain of the LDLR,${ }^{84}$ triggering its uptake and subsequent degradation. The net effect of this is to reduce the number of LDLRs available for clearing LDL from plasma at the cell surface. Parabiosis experiments in mice ${ }^{85}$ as well as administration of recombinant PCSK9 protein, ${ }^{86}$ have demonstrated that secreted PCSK9 is sufficient to initiate substantial degradation of the receptor. Interestingly, PCSK9 does not directly proteolyze the LDLR, and its catalytic activity is not required for its effect in promoting turnover of the LDLR..$^{87,88}$

In line with its role in coordinating LDL metabolism, PCSK9 expression is controlled by cellular cholesterol status via the action of SREBPs. Genetic manipulation of SREBP expression has substantial effects on PCSK9 mRNA levels in mice. ${ }^{41}$ The PCSK9 promoter contains a sterol response 


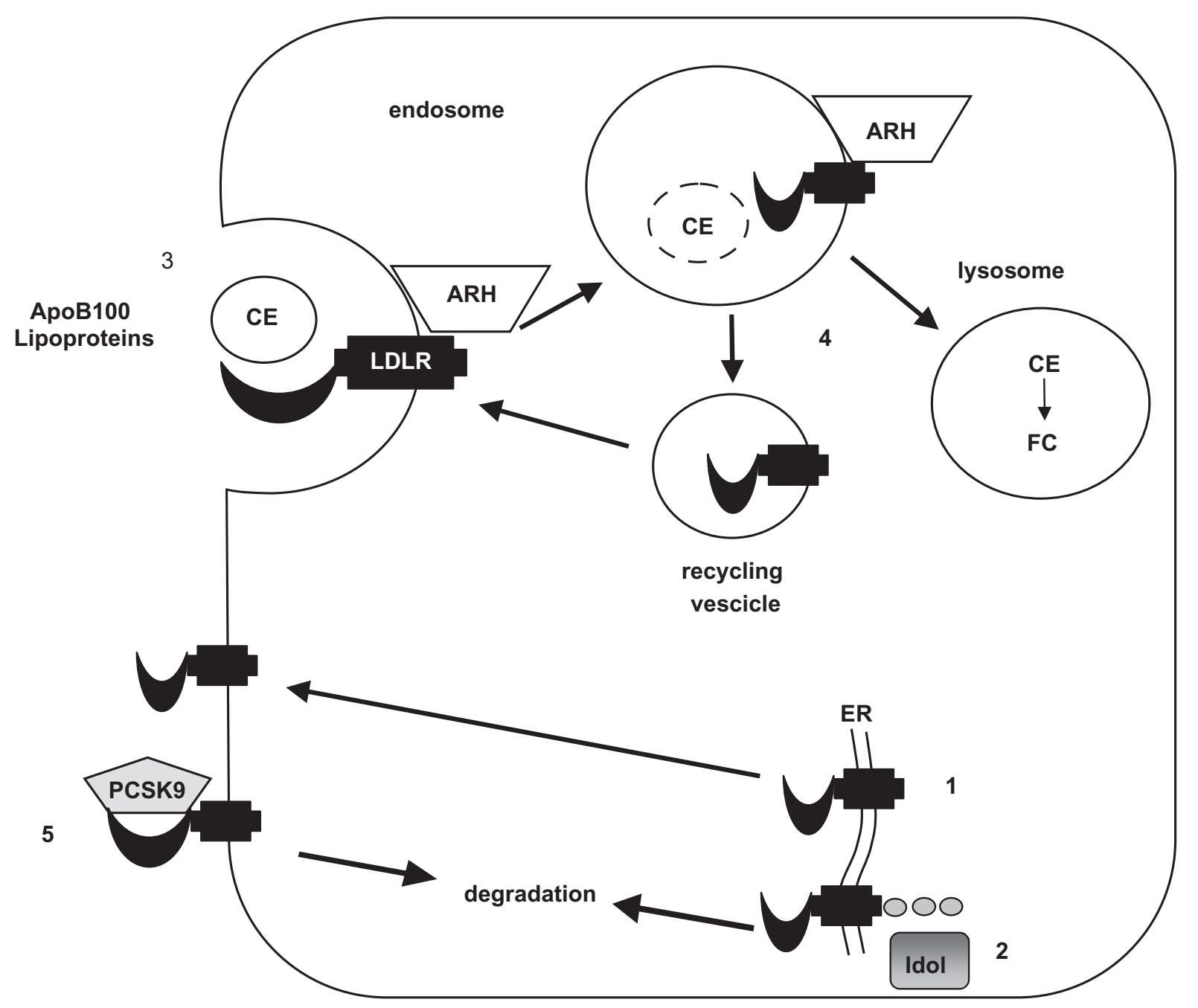

Figure 3 Regulation of low-density lipoprotein receptor (LDLR) trafficking and turnover by Idol,ARH and PCSK9. I) The LDLR is synthesized in the endoplasmic reticulum (ER) where it is transported to the plasma membrane. 2) In the presence of Idol the LDLR is ubiquitinated and degraded. 3) At the plasma membrane the LDLR is able to bind lipoproteins containing an apoBI00 or apoE ligand. Once bound this lipoprotein-receptor complex migrates into clathrin-coated pits and is internalized, a process that, in the liver, requires ARH. Endocytosed lipoproteins undergo release from the receptor and are degraded in the lysosome and cholesterol ester is hydrolyzed to free cholesterol that becomes membrane bound. 4) The receptor recycles back to the plasma membrane where it can bind additional lipoproteins. 5) PCSK9 can bind to the LDLR targeting the receptor for degradation once this complex is internalized.

Abbreviations: $\mathrm{ER}$, endoplasmic reticulum; $\mathrm{CE}$, cholesteryl ester; $\mathrm{FC}$, free cholesterol; $\mathrm{ARH}$, autosomal recessive hypercholesterolemia.

element (SRE) binding site, required for transcriptional activation by SREBPs ${ }^{89}$ SREBP-2 rather than $1 \mathrm{a}$ or $-1 \mathrm{c}$ is believed to be the major in vivo effector of PCSK9 transcription, as dietary cholesterol feeding decreases hepatic PCSK9 mRNA levels in mice. ${ }^{43,90}$ Statin treatment increases PCSK9 expression in HepG2 cells, as well as human hepatocytes, supporting the relevance of these findings to human LDL metabolism. ${ }^{91}$ Mice deficient in PCSK9 have much lower LDL cholesterol levels, and are more sensitive to cholesterol lowering by statins. ${ }^{92}$ These animals have a pronounced increase in LDLR protein upon statin treatment that is not normally observed in wild type mice. PCSK9 knockdown or inhibition would therefore be expected to synergistically augment the cholesterol lowering effect of statin therapy by preventing the normal induction of LDLR turnover by these drugs.

\section{Current drugs that regulate LDL receptor activity and abundance}

\section{Statins}

Development of the statins was pursued with the goal of specifically lowering LDL cholesterol through upregulation of the LDLR. Studies showed that inhibiting cholesterol synthesis in liver resulted in an increase in $L D L R$ expression which was expected to lead to increased LDL clearance 
from plasma. ${ }^{93}$ These findings led to the search for a compound to inhibit cholesterol synthesis in humans. The first agent found to be a safe and effective inhibitor of HMG CoA reductase was a naturally occurring inhibitor isolated from fungi, compactin (lovastatin) ${ }^{94}$ Since that time approximately 10 other drugs of this class have been developed and approved for use in humans, becoming the most prescribed medications worldwide.

Statins act by inhibiting 3-hydroxy-3-methylglutaryl Co enzyme A reductase, the enzyme that catalyzes the rate limiting step in cholesterol biosynthesis. Hepatic LDLR is exquisitely sensitive to changes in intracellular cholesterol content. In response to statin treatment which reduces cellular cholesterol by inhibiting cholesterol synthesis, hepatocytes upregulate LDLR activity to maintain an adequate supply of cholesterol. Induction of LDLR activity is widely believed to be a major mechanism by which statin treatment reduces the levels of circulating atherogenic LDL cholesterol in humans. Kinetic studies have demonstrated that statin treatment stimulates receptor-mediated clearance of $\mathrm{LDL}^{95,96}$ as well as LDL precursors (VLDL, IDL) from plasma. ${ }^{97}$ While statins also act to decrease cholesterol production, the cholesterol lowering ability of these drugs is greatly impaired in FH homozygotes that have a complete lack of LDLR activity when compared to heterozygotes or normal subjects. ${ }^{98,99}$ The upregulation of hepatic LDLR induced by statin treatment is believed to be caused by increased processing of sterol regulatory element binding protein 2 (SREBP-2), a transcriptional activator that plays a pivotal role in the maintenance of cholesterol homeostasis.

It should be noted that although statins have profound effects on hepatic LDLR mRNA levels and activity, they seem to have little impact on steady state LDLR protein levels in normal animals. As has been suggested, ${ }^{100}$ this paradox might be explained by increased cycling of the receptor. It is estimated that each LDLR molecule may be recycled back to the cell surface as many as 150 times. ${ }^{101}$ An increased rate of cycling between the cell surface and endosomal compartments would be predicted to remove a greater amount of LDL cholesterol from the circulation. However, in light of recent advances made in the regulation and role of PCSK9 in governing LDLR levels, ${ }^{92}$ we can now formulate an alternative hypothesis. Perhaps the actual rate of receptor cycling is not affected, but the flux of receptors from synthesis, to the cell surface, to intracellular delivery is increased (ie more receptors are made, more internalize and release cholesterol, and more are degraded at any given time). Cholesterol depletion caused by statin treatment activates SREBP-2 which simultaneously increases transcription of LDLR as well as PCSK9, a protein capable of promoting LDLR degradation. As more LDLR is synthesized and enters the pool, more can be degraded via PCSK9. The net effect may be increased delivery of LDL cholesterol to the liver. In support of this theory, cholesterol biosynthesis inhibitors such as statins and zaragozic acid A, increase the rate of LDLR synthesis, yet dramatically shorten the half-life of the LDLR protein. ${ }^{100}$ We postulate that increased flux of LDLR protein may be an important factor in statin-mediated cholesterol lowering. Further work is needed to determine whether increased receptor cycling or flux is occurring, and what roles PCSK9, Idol and ARH might play.

\section{Cholesterol absorption inhibitors}

Cholesterol absorption inhibitors include ezetimibe and phytosterols. Ezetimibe is an inhibitor of the cholesterol transporter, Niemann-Pick type C1-like 1 protein (NPC1L1). ${ }^{102}$ NPC1L1 is located on the luminal face of the enterocyte and is responsible for absorption of the majority of cholesterol by the small intestine. Inhibition of NPC1L1 in the small intestine leads to reduced delivery of dietary cholesterol to liver which, in turn, stimulates upregulation of the LDLR. Inhibition of NPC1L1 with ezetimibe has been shown to reduce cholesterol absorption by $54 \%$ when compared to placebo. ${ }^{103}$ This has been associated with a decrease in LDL cholesterol levels of $18 \%$ alone and by $53 \%$ when used in combination with a statin $^{104}$ since these drugs act to lower LDL levels through complimentary mechanisms.

Phytosterols are plant-derived sterols that are absorbed by the small intestine but rapidly excreted by the liver in bile. ${ }^{105}$ In the small intestine phytosterols can compete with cholesterol for absorption resulting in reduced cholesterol absorption. The net result is reduced delivery of dietary and biliary cholesterol to liver which, in turn, upregulates the LDLR.

\section{Fibrates}

Fibrates were initially approved for use in reducing high triglyceride levels associated with primary hypertriglyceridemia and mixed dyslipidemia. These drugs were shown to be effective in promoting lipolysis of triglyceride which was eventually shown to be due to activation of the nuclear receptor PPAR- $\alpha{ }^{106}$ Activation of PPAR- $\alpha$ promotes transcription of lipoprotein lipase and apoC-II while repressing expression of apoC-III, a lipase and LDLR inhibitor. Despite large effects on reducing apoC-III levels, fibrates and other PPAR- $\alpha$ have modest effects in reducing LDL cholesterol 
levels. ${ }^{107}$ Not surprisingly, most of the benefit of fibrates on reducing cardiovascular risk has been attributed to their effects on lowering plasma triglyceride levels and increasing HDL levels.

\section{Drugs under development to regulate LDL receptor activity and abundance CETP inhibitors}

Although initially designed as a means to increase HDL cholesterol levels, CETP inhibitors were serendipitously found to be effective in reducing LDL cholesterol levels in a dose-dependent manner. ${ }^{108}$ The reason for this is not completely known but may relate to a change in the delivery of cholesterol to the liver on plasma lipoproteins. Cholesterol delivered to the liver on HDL is thought to be targeted for biliary excretion whereas delivery on LDL is thought to enter the cholesterol regulatory pool where it can be re-secreted on VLDL. ${ }^{109}$ Since CETP inhibition enhances cholesterol delivery to the liver on HDL and reduces delivery on LDL the liver should respond by sensing less cholesterol and upregulating LDLR. In vivo studies support this model since treatment with a CETP inhibitor was associated with enhanced LDL clearance from plasma, presumably due to upregulation of LDLRs resulting from reduced LDL cholesterol delivery to liver. ${ }^{110}$

\section{Thyroid receptor- $\beta$ agonism}

As previously mentioned, thyroid hormone has long been known to have beneficial effects on plasma lipoproteins, particularly in regards to reducing LDL cholesterol due to direct effects on promoting LDLR transcription. Administration of thyroid hormone to patients with hypothyroidism has been associated with enhanced LDL clearance from plasma in patients with due to upregulation of the LDLR. ${ }^{11}$ However, due to other metabolic effects, particularly the effect of increasing heart rate, its use as a lipid lowering agent in the general population is not feasible. The discovery that there are two thyroid hormone receptor subtypes, $\alpha$ and $\beta$, that differentially affect heart rate and LDL cholesterol levels ${ }^{112}$ opened the door for development of specific agonists that could activate thyroid hormone receptor $\beta$, which is responsible for LDL cholesterol lowering, while leaving thyroid receptor $\alpha$, which stimulates heart rate, unaltered. Eprotirome/KB2115 is one such agonist. Initial studies have shown that the compound can safely be used to lower LDL cholesterol levels alone and in combination with statins. ${ }^{113}$

\section{RNA interference of PCSK9}

Small interfering RNAs (siRNA) are short double stranded RNAs that can be used to selectively silence target genes by binding to their messenger RNA in a sequence-specific manner. siRNAs utilize endogenous microRNA pathways to mediate degradation of the target message, thereby reducing synthesis of the corresponding protein product. A siRNA designed to target PCKS9 is being developed being designed for the treatment of hyperlipidemia. siRNA mediated degradation of PCSK9 mRNA would be expected to reduce circulating PCSK9 levels leading to reduced LDLR turnover. In animal models PCSK9 knockdown reduced PCSK 9 mRNA levels by approximately $50 \%$ with up to $70 \%$ reduction in LDL cholesterol levels. ${ }^{114}$

\section{Antisense oligonucleotides directed against apoC-III}

Antisense oligonucleotides (ASO) are chemically modified short single-stranded DNA or RNA molecules whose sequence is complementary to the mRNA of a target message. Unlike siRNAs, the single stranded ASOs are designed to bind tightly to the target message mediating its degradation via an RNAse-H dependent mechanism, resulting in decreased translation of the protein product. ${ }^{115}$ An ASO directed against apoC-III is currently in preclinical development. This drug is designed to reduce plasma levels of apoC-III, an endogenous inhibitor of lipoprotein lipase activity. Knockdown of ApoC-III would therefore act to potently reduce plasma triglycerides via increased lipolysis. In addition, Apo C-III is thought to interact with the LDLR and prevent binding of apoB on LDL. The benefit of reducing apoC-III levels would be enhanced binding of LDL to LDLRs, resulting in further LDL cholesterol lowering.

\section{Conclusion}

The discovery of the LDL receptor has led to some of the most beneficial therapies designed to reduce the risk of cardiovascular disease. These treatments have primarily targeted the cholesterol synthetic pathway leading to reduced intracellular cholesterol levels that, in turn, result in upregulation of LDLR expression and activity. The net result is enhanced clearance of LDL from plasma and reduced LDL levels. Recent advances in the regulation of LDLR turnover provide additional mechanisms that can be exploited to develop novel therapies designed to enhance LDLR activity. Recent evidence suggests that reducing LDL to below $70 \mathrm{mg} / \mathrm{dL}$ is associated with the regression of atherosclerosis. These therapies would complement existing therapies resulting 
in unprecedented degrees of LDL lowering that would be expected to reverse atherosclerosis further reducing the incidence of cardiovascular disease.

\section{Disclosures}

The authors declare no conflicts of interest.

\section{References}

1. Warner GJ, Stoudt G, Bamberger M, Johnson WJ, Rothblat GH. Cell toxicity induced by inhibition of acyl coenzyme A:cholesterol acyltransferase and accumulation of unesterified cholesterol. J Biol Chem. 1995;270(11):5772-5778.

2. Brown MS, Goldstein JL. Sterol regulatory element binding proteins (SREBPs): controllers of lipid synthesis and cellular uptake. Nutr Rev. 1998;56(2 Pt 2):S1-S3; discussion S54-S75.

3. Slater HR, McKinney L, Packard CJ, Shepherd J. Contribution of the receptor pathway to low density lipoprotein catabolism in humans. New methods for quantitation. Arteriosclerosis. 1984;4(6):604-613.

4. Brown MS, Goldstein JL. Cholesterol feedback: from Schoenheimer's bottle to Scap's MELADL. J Lipid Res. 2009;50(Suppl):S15-S27.

5. Goldstein JL, Brown MS. The LDL receptor. Arterioscler Thromb Vasc Biol. 2009;29(4):431-438.

6. Hobbs HH, Brown MS, Goldstein JL. Molecular genetics of the LDL receptor gene in familial hypercholesterolemia. Hum Mutat. 1992;1(6):445-466.

7. Muller C. Angina pectoris in heriditary xanthomatosis. Arch Intern Med. 1939;64:675-700.

8. Goldstein JL, Brown MS. Familial hypercholesterolemia: identification of a defect in the regulation of 3-hydroxy-3-methylglutaryl coenzyme A reductase activity associated with overproduction of cholesterol. Proc Natl Acad Sci U S A. 1973;70(10):2804-2808.

9. Brown MS, Goldstein JL. Familial hypercholesterolemia: defective binding of lipoproteins to cultured fibroblasts associated with impaired regulation of 3-hydroxy-3-methylglutaryl coenzyme A reductase activity. Proc Natl Acad Sci U S A. 1974;71(3):788-792.

10. Brown MS, Goldstein JL. Regulation of the activity of the low density lipoprotein receptor in human fibroblasts. Cell. 1975;6(3): 307-316.

11. Schneider WJ, Beisiegel U, Goldstein JL, Brown MS. Purification of the low density lipoprotein receptor, an acidic glycoprotein of 164,000 molecular weight. J Biol Chem. 1982;257(5):2664-2673.

12. Sudhof TC, Goldstein JL, Brown MS, Russell DW. The LDL receptor gene: a mosaic of exons shared with different proteins. Science. 1985; 228(4701):815-822.

13. Brown MS, Herz J, Goldstein JL. LDL-receptor structure. Calcium cages, acid baths and recycling receptors. Nature. 1997;388(6643): 629-630.

14. Kolansky DM, Cuchel M, Clark BJ, et al. Longitudinal evaluation and assessment of cardiovascular disease in patients with homozygous familial hypercholesterolemia. Am J Cardiol. 2008;102(11):1438-1443.

15. Shireman R, Kilgore LL, Fisher WR. Solubilization of apolipoprotein $B$ and its specific binding by the cellular receptor for low density lipoprotein. Proc Natl Acad Sci U S A. 1977;74(11):5150-5154.

16. Mahley RW, Innerarity TL, Weisgraber KH, Fry DL. Canine hyperlipoproteinemia and atherosclerosis. Accumulation of lipid by aortic medial cells in vivo and in vitro. Am J Pathol. 1977;87(1):205-226.

17. Yamamoto T, Davis CG, Brown MS, et al. The human LDL receptor: a cysteine-rich protein with multiple Alu sequences in its mRNA. Cell. 1984;39(1):27-38.

18. Mahley RW, Weisgraber KH, Melchior GW, Innerarity TL, Holcombe KS. Inhibition of receptor-mediated clearance of lysine and arginine-modified lipoproteins from the plasma of rats and monkeys. Proc Natl Acad Sci U S A. 1980;77(1):225-229.
19. Innerarity TL, Friedlander EJ, Rall SC Jr, Weisgraber KH, Mahley RW. The receptor-binding domain of human apolipoprotein E. Binding of apolipoprotein E fragments. J Biol Chem. 1983;258(20): 12341-12347.

20. Kita T, Brown MS, Watanabe Y, Goldstein JL. Deficiency of low density lipoprotein receptors in liver and adrenal gland of the WHHL rabbit, an animal model of familial hypercholesterolemia. Proc Natl Acad Sci US A. 1981;78(4):2268-2272.

21. Russell DW, Schneider WJ, Yamamoto T, et al. Domain map of the LDL receptor: sequence homology with the epidermal growth factor precursor. Cell. 1984;37(2):577-585.

22. Jeon H, Meng W, Takagi J, Eck MJ, Springer TA, Blacklow SC. Implications for familial hypercholesterolemia from the structure of the LDL receptor YWTD-EGF domain pair. Nat Struct Biol. 2001;8(6):499-504.

23. Rudenko G, Henry L, Henderson K, et al. Structure of the LDL receptor extracellular domain at endosomal $\mathrm{pH}$. Science. 2002;298(5602):2353-2358.

24. Kozarsky K, Kingsley D, Krieger M. Use of a mutant cell line to study the kinetics and function of O-linked glycosylation of low density lipoprotein receptors. Proc Natl Acad Sci U S A. 1988;85(12): 4335-4339.

25. Davis CG, Elhammer A, Russell DW, et al. Deletion of clustered O-linked carbohydrates does not impair function of low density lipoprotein receptor in transfected fibroblasts. $J$ Biol Chem. 1986;261(6): 2828-2838.

26. Lehrman MA, Schneider WJ, Sudhof TC, et al. Mutation in LDL receptor: Alu-Alu recombination deletes exons encoding transmembrane and cytoplasmic domains. Science. 1985;227(4683):140-146.

27. Garcia CK, Wilund K, Arca M, et al. Autosomal recessive hypercholesterolemia caused by mutations in a putative LDL receptor adaptor protein. Science. 2001;292(5520):1394-1398.

28. Koivisto UM, Hubbard AL, Mellman I. A novel cellular phenotype for familial hypercholesterolemia due to a defect in polarized targeting of LDL receptor. Cell. 2001;105(5):575-585.

29. Chatterton JE, Phillips ML, Curtiss LK, et al. Immunoelectron microscopy of low density lipoproteins yields a ribbon and bow model for the conformation of apolipoprotein B on the lipoprotein surface. $J$ Lipid Res. 1995;36(9):2027-2037.

30. Packard CJ, Munro A, Lorimer AR, Gotto AM, Shepherd J. Metabolism of apolipoprotein B in large triglyceride-rich very low density lipoproteins of normal and hypertriglyceridemic subjects. J Clin Invest. 1984;74(6):2178-2192.

31. Barter PJ, Brewer HB Jr, Chapman MJ, Hennekens CH, Rader DJ, Tall AR. Cholesteryl ester transfer protein: a novel target for raising HDL and inhibiting atherosclerosis. Arterioscler Thromb Vasc Biol. 2003;23(2):160-167.

32. Krul ES, Tikkanen MJ, Cole TG, Davie JM, Schonfeld G. Roles of apolipoproteins $\mathrm{B}$ and $\mathrm{E}$ in the cellular binding of very low density lipoproteins. J Clin Invest. 1985;75(2):361-369.

33. Zaiou M, Arnold KS, Newhouse YM, et al. Apolipoprotein E;-low density lipoprotein receptor interaction. Influences of basic residue and amphipathic alpha-helix organization in the ligand. J Lipid Res. 2000;41(7):1087-1095.

34. Vega GL, Grundy SM. In vivo evidence for reduced binding of low density lipoproteins to receptors as a cause of primary moderate hypercholesterolemia. J Clin Invest. 1986;78(5):1410-1414.

35. Lamon-Fava S, Diffenderfer MR, Barrett PH, et al. Effects of different doses of atorvastatin on human apolipoprotein B-100, B-48, and A-I metabolism. J Lipid Res. 2007;48(8):1746-1753.

36. Radhakrishnan A, Goldstein JL, McDonald JG, Brown MS. Switchlike control of SREBP-2 transport triggered by small changes in ER cholesterol: a delicate balance. Cell Metab. 2008;8(6): $512-521$.

37. Brown MS, Goldstein JL. The SREBP pathway: regulation of cholesterol metabolism by proteolysis of a membrane-bound transcription factor. Cell. 1997;89(3):331-340. 
38. Sato R, Yang J, Wang X, et al. Assignment of the membrane attachment, DNA binding, and transcriptional activation domains of sterol regulatory element-binding protein-1 (SREBP-1). J Biol Chem. 1994;269(25):17267-17273.

39. Yokoyama C, Wang X, Briggs MR, and colleagues SREBP-1, a basic-helix-loop-helix-leucine zipper protein that controls transcription of the low density lipoprotein receptor gene. Cell. 1993;75(1): 187-197.

40. Hua X, Yokoyama C, Wu J, et al. SREBP-2, a second basic-helixloop-helix-leucine zipper protein that stimulates transcription by binding to a sterol regulatory element. Proc Natl Acad Sci U S A. 1993;90(24):11603-11607.

41. Horton JD, Shah NA, Warrington JA, et al. Combined analysis of oligonucleotide microarray data from transgenic and knockout mice identifies direct SREBP target genes. Proc Natl Acad Sci U S A. 2003;100(21):12027-12032.

42. Shimano H. Sterol regulatory element-binding proteins (SREBPs): transcriptional regulators of lipid synthetic genes. Prog Lipid Res. 2001;40(6):439-452.

43. Shimano H, Shimomura I, Hammer RE, et al. Elevated levels of SREBP-2 and cholesterol synthesis in livers of mice homozygous for a targeted disruption of the SREBP-1 gene. J Clin Invest. 1997;100(8):2115-2124.

44. Natesampillai S, Fernandez-Zapico ME, Urrutia R, Veldhuis JD A novel functional interaction between the Sp1-like protein KLF13 and SREBP-Sp1 activation complex underlies regulation of low density lipoprotein receptor promoter function. J Biol Chem. 2006;281(6): 3040-3047.

45. Cohen JC, Kimmel M, Polanski A, Hobbs HH. Molecular mechanisms of autosomal recessive hypercholesterolemia. Curr Opin Lipidol. 2003;14(2):121-127.

46. Wilund KR, Yi M, Campagna F, et al. Molecular mechanisms of autosomal recessive hypercholesterolemia. Hum Mol Genet. 2002;11(24):3019-3030.

47. Khachadurian AK, Uthman SM. Experiences with the homozygous cases of familial hypercholesterolemia. A report of 52 patients. Nutr Metab. 1973;15(1):132-140.

48. Arca M, Zuliani G, Wilund K, et al. Autosomal recessive hypercholesterolaemia in Sardinia, Italy, and mutations in ARH: a clinical and molecular genetic analysis. Lancet. 2002;359(9309): 841-847.

49. Zuliani G, Arca M, Signore A, et al. Characterization of a new form of inherited hypercholesterolemia: familial recessive hypercholesterolemia. Arterioscler Thromb Vasc Biol. 1999;19(3):802-809.

50. Harada-Shiba M, Tajima S, Yokoyama S, et al. Siblings with normal LDL receptor activity and severe hypercholesterolemia. Arterioscler Thromb. 1992;12(9):1071-1078.

51. He G, Gupta S, Yi M, Michaely P, Hobbs HH, Cohen JC. ARH is a modular adaptor protein that interacts with the LDL receptor, clathrin, and AP-2. J Biol Chem. 2002;277(46):44044-44049.

52. Jones C, Hammer RE, Li WP, Cohen JC, Hobbs HH, Herz J. Normal sorting but defective endocytosis of the low density lipoprotein receptor in mice with autosomal recessive hypercholesterolemia. J Biol Chem. 2003;278(31):29024-29030.

53. Garuti R, Jones C, Li WP, et al. The modular adaptor protein autosomal recessive hypercholesterolemia $(\mathrm{ARH})$ promotes low density lipoprotein receptor clustering into clathrin-coated pits. J Biol Chem. 2005;280(49):40996-41004.

54. Maurer ME, Cooper JA. The adaptor protein Dab2 sorts LDL receptors into coated pits independently of AP-2 and ARH. J Cell Sci. 2006; 119(Pt 20):4235-4246.

55. Zelcer N, Hong C, Boyadjian R, Tontonoz P. LXR regulates cholesterol uptake through Idol-dependent ubiquitination of the LDL receptor. Science. 2009;325(5936):100-104.

56. Olsson PA, Korhonen L, Mercer EA, Lindholm D. MIR is a novel ERM-like protein that interacts with myosin regulatory light chain and inhibits neurite outgrowth. J Biol Chem. 1999;274(51):36288-36292.
57. Chait A, Bierman EL, Albers JJ. Regulatory role of triiodothyronine in the degradation of low density lipoprotein by cultured human skin fibroblasts. J Clin Endocrinol Metab. 1979;48(5):887-889.

58. Bhattacharya S, Balasubramaniam S, Simons LA. Regulation of low-density-lipoprotein metabolism in the rat. Biochem J. 1986;234(2):493-496.

59. Thompson GR, Soutar AK, Spengel FA, et al. Defects of receptormediated low density lipoprotein catabolism in homozygous familial hypercholesterolemia and hypothyroidism in vivo. Proc Natl Acad Sci U S A. 1981;78(4):2591-2595.

60. Staels B, Van Tol A, Chan L, Will H, Verhoeven G, Auwerx J. Alterations in thyroid status modulate apolipoprotein, hepatic triglyceride lipase, and low density lipoprotein receptor in rats. Endocrinology. 1990;127(3):1144-1152.

61. Ness GC, Pendleton LC, Li YC, Chiang JY. Effect of thyroid hormone on hepatic cholesterol 7 alpha hydroxylase, LDL receptor, HMG-CoA reductase, farnesyl pyrophosphate synthetase and apolipoprotein A-I mRNA levels in hypophysectomized rats. Biochem Biophys Res Commun. 1990;172(3):1150-1156.

62. Ness GC, Lopez D, Chambers CM, et al. Effects of L-triiodothyronine and the thyromimetic L-94901 on serum lipoprotein levels and hepatic low-density lipoprotein receptor, 3-hydroxy-3-methylglutaryl coenzyme A reductase, and apo A-I gene expression. Biochem Pharmacol. 1998;56(1):121-129.

63. Ness GC, Zhao Z. Thyroid hormone rapidly induces hepatic LDL receptor mRNA levels in hypophysectomized rats. Arch Biochem Biophys. 1994;315(1):199-202.

64. Ness GC, Lopez D. Transcriptional regulation of rat hepatic low-density lipoprotein receptor and cholesterol 7 alpha hydroxylase by thyroid hormone. Arch Biochem Biophys. 1995;323(2):404-408.

65. Bakker O, Hudig F, Meijssen S, Wiersinga WM. Effects of triiodothyronine and amiodarone on the promoter of the human LDL receptor gene. Biochem Biophys Res Commun. 1998;249(2):517-521.

66. Shin DJ, Osborne TF. Thyroid hormone regulation and cholesterol metabolism are connected through Sterol Regulatory Element-Binding Protein-2 (SREBP-2). J Biol Chem. 2003;278(36):34114-34118.

67. Lopez D, Abisambra Socarras JF, Bedi M, Ness GC. Activation of the hepatic LDL receptor promoter by thyroid hormone. Biochim Biophys Acta. 2007;1771(9):1216-1225

68. Marcel YL, Hogue M, Weech PK, Davignon J, Milne RW. Expression of apolipoprotein B epitopes in lipoproteins. Relationship to conformation and function. Arteriosclerosis. 1988;8(6):832-844.

69. Franceschini G, Bernini F, Michelagnoli S, et al. Lipoprotein changes and increased affinity of LDL for their receptors after acipimox treatment in hypertriglyceridemia. Atherosclerosis. 1990;81(1):41-49.

70. Boren J, Lee I, Zhu W, et al. Identification of the low density lipoprotein receptor-binding site in apolipoprotein B100 and the modulation of its binding activity by the carboxyl terminus in familial defective apo-B100. J Clin Invest. 1998;101(5):1084-1093.

71. Soria LF, Ludwig EH, Clarke HR, et al. Association between a specific apolipoprotein B mutation and familial defective apolipoprotein B-100. Proc Natl Acad Sci US A. 1989;86(2):587-591.

72. Clavey V, Lestavel-Delattre S, Copin C, Bard JM, Fruchart JC. Modulation of lipoprotein B binding to the LDL receptor by exogenous lipids and apolipoproteins CI, CII, CIII, and E. Arterioscler Thromb Vasc Biol. 1995;15(7):963-971.

73. Bradley WA, Hwang SL, Karlin JB, et al. Low-density lipoprotein receptor binding determinants switch from apolipoprotein $\mathrm{E}$ to apolipoprotein $\mathrm{B}$ during conversion of hypertriglyceridemic very-low-density lipoprotein to low-density lipoproteins. J Biol Chem. 1984;259(23):14728-14735.

74. Millar JS, Maugeais C, Fuki IV, Rader DJ. Normal production rate of apolipoprotein B in LDL receptor-deficient mice. Arterioscler Thromb Vasc Biol. 2002;22(6):989-994.

75. Millar JS, Maugeais C, Ikewaki K, et al. Complete deficiency of the low-density lipoprotein receptor is associated with increased apolipoprotein B-100 production. Arterioscler Thromb Vasc Biol. 2005;25(3):560-565. 
76. Twisk J, Gillian-Daniel DL, Tebon A, et al. The role of the LDL receptor in apolipoprotein B secretion. $J$ Clin Invest. 2000;105(4):521-532.

77. Gillian-Daniel DL, Bates PW, Tebon A, Attie AD. Endoplasmic reticulum localization of the low density lipoprotein receptor mediates presecretory degradation of apolipoprotein B. Proc Natl Acad Sci US A. 2002;99(7):4337-4342.

78. Zheng C, Khoo C, Ikewaki K, Sacks FM. Rapid turnover of apolipoprotein $\mathrm{C}$-III-containing triglyceride-rich lipoproteins contributing to the formation of LDL subfractions. J Lipid Res. 2007;48(5): 1190-1203.

79. Gangabadage CS, Zdunek J, Tessari M, et al. Structure and dynamics of human apolipoprotein CIII. J Biol Chem. 2008;283(25): 17416-17427.

80. Pollin TI, Damcott CM, Shen H, et al. A null mutation in human APOC3 confers a favorable plasma lipid profile and apparent cardioprotection. Science. 2008;322(5908):1702-1705.

81. Abifadel M, Varret M, Rabes JP, et al. Mutations in PCSK9 cause autosomal dominant hypercholesterolemia. Nat Genet. 2003;34(2):154-156.

82. Cohen J, Pertsemlidis A, Kotowski IK, et al. Low LDL cholesterol in individuals of African descent resulting from frequent nonsense mutations in PCSK9. Nat Genet. 2005;37(2):161-165.

83. Seidah NG, Benjannet S, Wickham L, et al. The secretory proprotein convertase neural apoptosis-regulated convertase 1 (NARC-1): liver regeneration and neuronal differentiation. Proc Natl Acad Sci US A. 2003;100(3):928-933.

84. Zhang DW, Lagace TA, Garuti R, et al. Binding of proprotein convertase subtilisin/kexin type 9 to epidermal growth factor-like repeat A of low density lipoprotein receptor decreases receptor recycling and increases degradation. J Biol Chem. 2007;282(25):18602-18612.

85. Lagace TA, Curtis DE, Garuti R, et al. Secreted PCSK9 decreases the number of LDL receptors in hepatocytes and in livers of parabiotic mice. J Clin Invest. 2006;116(11):2995-3005.

86. Grefhorst A, McNutt MC, Lagace TA, Horton JD. Plasma PCSK9 preferentially reduces liver LDL receptors in mice. J Lipid Res. 2008;49(6):1303-1311.

87. Horton JD, Cohen JC, Hobbs HH. Molecular biology of PCSK9: its role in LDL metabolism. Trends Biochem Sci. 2007;32(2):71-77.

88. Li J, Tumanut C, Gavigan JA, et al. Secreted PCSK9 promotes LDL receptor degradation independently of proteolytic activity. Biochem $J$. 2007;406(2):203-207.

89. Jeong HJ, Lee HS, Kim KS, et al. Sterol-dependent regulation of proprotein convertase subtilisin/kexin type 9 expression by sterol-regulatory element binding protein-2. J Lipid Res. 2008;49(2):399-409.

90. Maxwell KN, Soccio RE, Duncan EM, Sehayek E, Breslow JL. Novel putative SREBP and LXR target genes identified by microarray analysis in liver of cholesterol-fed mice. J Lipid Res. 2003;44(11): 2109-2119.

91. Dubuc G, Chamberland A, Wassef H, et al. Statins upregulate PCSK9, the gene encoding the proprotein convertase neural apoptosis-regulated convertase-1 implicated in familial hypercholesterolemia. Arterioscler Thromb Vasc Biol. 2004;24(8):1454-1459.

92. Rashid S, Curtis DE, Garuti R, et al. Decreased plasma cholesterol and hypersensitivity to statins in mice lacking Pcsk9. Proc Natl Acad Sci U S A. 2005;102(15):5374-5379.

93. Endo A, Kuroda M, Tanzawa K. Competitive inhibition of 3-hydroxy3-methylglutaryl coenzyme A reductase by ML-236A and ML-236B fungal metabolites, having hypocholesterolemic activity. FEBS Lett. 1976;72(2):323-326.

94. Mabuchi H, Haba T, Tatami R, et al. Effect of an inhibitor of 3-hydroxy3-methyglutaryl coenzyme A reductase on serum lipoproteins and ubiquinone-10-levels in patients with familial hypercholesterolemia. N Engl J Med. 1981;305(9):478-482.

95. Bilheimer DW, Grundy SM, Brown MS, Goldstein JL. Mevinolin and colestipol stimulate receptor-mediated clearance of low density lipoprotein from plasma in familial hypercholesterolemia heterozygotes. Proc Natl Acad Sci U S A. 1983;80(13):4124-4128.
96. Bilheimer DW, Grundy SM, Brown MS, Goldstein JL. Mevinolin stimulates receptor-mediated clearance of low density lipoprotein from plasma in familial hypercholesterolemia heterozygotes. Trans Assoc Am Physicians. 1983;96:1-9.

97. Magot T, Malmendier CL, Ouguerram K, Lontie JF, Lutton C. In vivo effect of simvastatin on lipoprotein cholesteryl ester metabolism in normocholesterolemic volunteers. Clin Chim Acta. 1991;196(1):59-68.

98. Berglund L, Witztum JL, Galeano NF, et al. Three-fold effect of lovastatin treatment on low density lipoprotein metabolism in subjects with hyperlipidemia: increase in receptor activity, decrease in apoB production, and decrease in particle affinity for the receptor. Results from a novel triple-tracer approach. J Lipid Res. 1998;39(4):913-924.

99. Marais AD, Naoumova RP, Firth JC, et al. Decreased production of low density lipoprotein by atorvastatin after apheresis in homozygous familial hypercholesterolemia. $J$ Lipid Res. 1997;38(10):2071-2078.

100. Ness GC, Zhao Z, Lopez D. Inhibitors of cholesterol biosynthesis increase hepatic low-density lipoprotein receptor protein degradation. Arch Biochem Biophys. 1996;325(2):242-248.

101. Brown MS, Anderson RG, Goldstein JL. Recycling receptors: the round-trip itinerary of migrant membrane proteins. Cell. 1983;32(3):663-667.

102. Altmann SW, Davis HR, Jr., Zhu LJ, et al. Niemann-Pick C1 Like 1 protein is critical for intestinal cholesterol absorption. Science. 2004;303(5661):1201-1204.

103. Sudhop T, Lutjohann D, Kodal A, et al. Inhibition of intestinal cholesterol absorption by ezetimibe in humans. Circulation. 2002;106(15):1943-1948.

104. Kastelein JJ, Akdim F, Stroes ES, et al. Simvastatin with or without ezetimibe in familial hypercholesterolemia. $N$ Engl $J$ Med. 2008;358(14):1431-1443.

105. Balmer J, Zilversmit DB. Effects of dietary roughage on cholesterol absorption, cholesterol turnover and steroid excretion in the rat. J Nutr. 1974;104(10):1319-1328.

106. Schoonjans K, Staels B, Auwerx J. Role of the peroxisome proliferator-activated receptor (PPAR) in mediating the effects of fibrates and fatty acids on gene expression. J Lipid Res. 1996;37(5): 907-925.

107. Nissen SE, Nicholls SJ, Wolski K, et al. Effects of a potent and selective PPAR-alpha agonist in patients with atherogenic dyslipidemia or hypercholesterolemia: two randomized controlled trials. JAMA. 2007;297(12):1362-1373.

108. Krishna R, Anderson MS, Bergman AJ, et al. Effect of the cholesteryl ester transfer protein inhibitor, anacetrapib, on lipoproteins in patients with dyslipidaemia and on 24-h ambulatory blood pressure in healthy individuals: two double-blind, randomised placebo-controlled phase I studies. Lancet. 2007;370(9603):1907-1914.

109. Schwartz CC, Halloran LG, Vlahcevic ZR, Gregory DH, Swell L. Preferential utilization of free cholesterol from high-density lipoproteins for biliary cholesterol secretion in man. Science. 1978;200(4337): $62-64$.

110. Millar JS, Brousseau ME, Diffenderfer MR, et al. Effects of the cholesteryl ester transfer protein inhibitor torcetrapib on apolipoprotein B100 metabolism in humans. Arterioscler Thromb Vasc Biol. 2006;26(6):1350-1356.

111. Packard CJ, Shepherd J, Lindsay GM, Gaw A, Taskinen MR. Thyroid replacement therapy and its influence on postheparin plasma lipases and apolipoprotein-B metabolism in hypothyroidism. $J$ Clin Endocrinol Metab. 1993;76(5):1209-1216.

112. Grover GJ, Mellstrom K, Malm J. Development of the thyroid hormone receptor beta-subtype agonist KB-141: a strategy for body weight reduction and lipid lowering with minimal cardiac side effects. Cardiovasc Drug Rev. 2005;23(2):133-148.

113. Erion MD, Cable EE, Ito BR, et al. Targeting thyroid hormone receptor-beta agonists to the liver reduces cholesterol and triglycerides and improves the therapeutic index. Proc Natl Acad Sci U S A. 2007;104(39):15490-15495. 
114. Frank-Kamenetsky M, Grefhorst A, Anderson NN, et al. Therapeutic RNAi targeting PCSK9 acutely lowers plasma cholesterol in rodents and LDL cholesterol in nonhuman primates. Proc Natl Acad Sci USA. 2008;105(33):11915-11920.
115. Stein CA, Cohen JS. Oligodeoxynucleotides as inhibitors of gene expression: a review. Cancer Res. 1988;48(10):2659-2668.

\section{Publish your work in this journal}

The Journal of Receptor, Ligand and Channel Research is an international, peer-reviewed, open access, online journal. The journal welcomes laboratory and clinical findings in the fields of biological receptors, ligands, channel and signal transduction research including: receptors and signalling; ligands; transporters, pores and channels; binding and activation; receptor regulation; role of receptors in diseases and their treatment; molecular basis of membrane structure and functions; molecular models of membranes. The manuscript management system is completely online and includes a very quick and fair peer-review system. Visit http://www.dovepress.com/ testimonials.php to read real quotes from published authors. 